\title{
ON APPROXIMATE BOUNDS OF ZEROS OF POLYNOMIALS WITHIN AND ON THE UNIT DISC
}

\author{
S.Sripriy1 ${ }^{2}$, Ajeet Singh ${ }^{2}$, P.K.Raina ${ }^{3}$ \\ ${ }^{1}$ Department of Mathematics, Lingayas University, Faridabad, Haryana \\ ${ }^{2}$ Department of Mathematics, Lingayas University, Faridabad, Haryana \\ ${ }^{3}$ Department of Mathematics, Institute of Education, $J \& K$
}

\begin{abstract}
In this paper we obtain a result concerning the location of the zeros of a class of polynomials $p(z)=\sum_{j=0}^{n} a_{j} z^{j}, \quad a_{n} \neq 0$, where $a_{n}=1$ and $a_{j}{ }^{\prime} s$ are real and complex coefficient and numerically less than unity for $j=0,1, \ldots . ., n$. We obtain least upper bounds enclosing the zeros of polynomials as sharper bounds and compare these with the ones given by Affane-Aji et al [1] and others [5, 6, 9].
\end{abstract}

\section{INTRODUCTION}

Current interest of researchers is to study the location of zero of polynomials as such they have been writing extensively on the works based on Gauss [4] and Cauchy [2]. Numerous books and papers have been written in modern areas of digital signal processing, Communication theory, Control theory and Cryptography, to mention a few and since then there is a greater need for improving the bounds of the zeros of the polynomials. In this paper we show that if all the coefficient of class of polynomials are numerically less than unity. Then our method gives the sharper bounds as compared to the ones given by Affane-Aji et al [1]. Moreover, we show by way of examples that their estimated bounds over estimate our bounds in all the cases under present investigation.

The first result regarding the location of zeros of polynomials is due to Gauss whose result was improved by Cauchy [2] as given by the following theorem:

$$
\{z:|z|<\xi\} \subset\{z:|z|<1+A\} \text {, }
$$

Where $A=\max \left|a_{j}\right|, 0 \leq j \leq n-1$, and $\xi$ is a unique positive root of the real coefficient polynomial

$$
Q(x)=x^{n}-\left|a_{n-1}\right| x^{n-1}-\left|a_{n-2}\right| x^{n-2}---\left|a_{1}\right| x-\left|a_{0}\right| .
$$

C-Affane-Aji et al [1] have given the following general result of sharper bounds pertaining to the zeros of polynomials that also include all the bounds obtained by earlier authors $[5,6,9]$.

Theorem 2.1: All the zeros of polynomials

$$
p(z)=z^{n}+\sum_{j=0}^{n-1} a_{j} z^{j}
$$

Theorem 1: Let $p(z)=z^{n}+\sum_{j=0}^{n-1} a_{j} z^{j}$ be the complex

polynomial. Then all the zeros of $p(z)$ lie in the disc:

Lie in disc:

$$
\{z:|z|<\xi\} \subset\left\{z:|z|<1+\delta_{k}\right\} \subset\left\{z:|z|<1+\delta_{k-1}\right\} \ldots . . \subset\left\{z:|z|<1+\delta_{1}\right\} \subset\{z:|z|<1+A\}
$$

Where $\delta_{k}$ is the unique positive root of the characteristic equation:

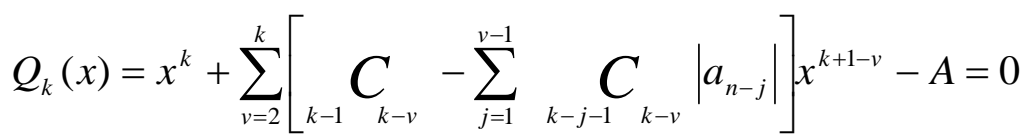


For $k>n$ or $\leq n$

Where $A=\max \left|a_{j}\right|, a_{j}=0, j<0$,

$0 \leq j \leq n-1 \& n C_{r}=0$, if $n<r<0$.

We note here that ${ }^{n} C_{r}=\frac{n !}{(n-r) !(r) !}$ and may as will be denoted by $\left(\begin{array}{l}n \\ r\end{array}\right)$

Result 1: For $\mathrm{k}=2$ from above theorem, the bound and characteristic Eq. (2.1.2) turn out to be:

$$
\left\{z:|z|<1+\delta_{2}\right\} \subset\{z:|z| \leq 1+A\},
$$

Where $\delta_{2}$ is the unique positive root of the respective characteristic equation

$$
Q_{2}(x)=x^{2}+\left(1-\left|a_{n-1}\right|\right) x-A=0
$$

The above equations coincide with the bounds given by joyal et al [6].

Result 2: For $k=3$, equation (2.1.1) gives

$$
\left\{z:|z|<1+\delta_{3}\right\} \subset\left\{z:|z|<1+\delta_{2}\right\} \subset\{z:|z|<1+A\},
$$

Where $\delta_{3}$ is unique positive root of the characteristic equation (2.1.2) is

$Q_{3}(x)=x^{3}+\left(2-\left|a_{n-1}\right|\right) x^{2}+\left(1-\left|a_{n-1}\right|-\left|a_{n-2}\right|\right) x-A=0$,

Which coincides with the bounds given by Sun [10]

Result 3 For $\mathrm{k}=4$, equation (2.1.1) turns out and to be the $\operatorname{disc}$

$$
\{z:|z|<\xi\} \subset\left\{z:|z|<1+\delta_{4}\right\} \subset\left\{z:|z|<1+\delta_{3}\right\} \subset\left\{z:|z|<1+\delta_{2}\right\} \subset\{z:|z|<1+A\}
$$

Where $\delta_{4}$ is the unique positive root of characteristic

$$
\begin{aligned}
& \text { equation } \\
& Q_{4}(x)=x^{4}+\left(3-\left|a_{n-1}\right|\right) x^{3}+\left(3-2\left|a_{n-1}\right|-\left|a_{n-2}\right|\right) x^{2}+\left(1-\left|a_{n-1}\right|-\left|a_{n-2}\right|-\left|a_{n-3}\right|\right) x-A=\sum_{j=0}^{n-1} a_{j} z^{j},
\end{aligned}
$$

Which coincides with the bounds given by Jain [5]
We, thus are able to show that this result provides a tool for obtaining sharper bounds for the location of the zeros of a class of polynomials with the real or complex co-efficient each of which is numerically less than unity based on the following remarks on the bounds of the zeros of polynomial:

\section{BOUNDS ON ZEROS}

The search for the real zeros of a polynomial function can be reduced if bounds on the zeros are found. A number $M$ is bound on the root of a polynomial if every zero lies between $\mathrm{M}$ and $\mathrm{M}$ inclusive. That is $\mathrm{M}$ is a bound to the zeros of a polynomial $\mathrm{f}$ if $-M \leq$ any zerosof $f \leq M$.

\section{Lemma 1:}

Let $\mathrm{f}$ denote a polynomial function whose leading coefficient is 1 as given by.

$$
f(z)=z^{n}+a_{n-1} z^{n-1}+\ldots \ldots .+a_{1} z+a_{0}
$$

A bound $\mathrm{M}$ on the zero of $\mathrm{f}$ is the smaller of the two numbers

$$
\max \left\{1, \sum_{j=0}^{n-1}\left|a_{j}\right|\right\}, \quad 1+\operatorname{Max}\left\{a_{j} \mid\right\}, j=0,1, \ldots, n-1
$$

Where max means \{\} "Choose largest entry in \{\}$"$. The bounds on the zeros of a polynomial provides good choice for setting zero min and zero maximum ( $\mathrm{Z}$ min and $\mathrm{Z}$ max) of the viewing of an angular region.

We note here that we are interested in finding the unknown radius $\xi$ to determine the smaller circular bound containing the zeros of the polynomials.

In this paper, using above Lemma 1 , we replace $1+\mathrm{A}$ where $\mathrm{A}=\operatorname{Max}\left|a_{j}\right| 0 \leq j \leq n-1$ in above theorem [2.1] by $\mathrm{B}=$ $\operatorname{Max}\{1, \mathrm{D}\}$ where $D=\sum_{j=0}^{n-1}\left|a_{j}\right|$.

In order to show that our estimate of bounds are the least upper bounds than obtained by $C$ Affane-Aji, we derive

\section{Theorem 3}

All the zeros of polynomial 
Lie in the disc:

$$
\{z \mid<\xi\} \subset\left\{z:|z|<\eta_{k}\right\} \subset\left\{z:|z|<\eta_{k-1}\right\} \ldots \subset\left\{z:|z|<\eta_{2}\right\} \subset\{z:|z|<\beta\},
$$

Where $\eta_{k}$ is the unique positive root of the characteristic equation

$$
Q_{k}(x)=x^{k}+\sum_{v=2}^{k}\left[C_{k-1}-\sum_{j=1}^{x-1} C_{k-j-1}\left|a_{n-j}\right|\right] x^{k+1-v}-D=0
$$

For $k>n$ or $\leq n$,

$$
\text { Where } B=\max \{1, D\} \text { subject to } D=\sum_{j=0}^{n-1}\left|a_{j}\right| \text {. }
$$

Here we note that for the class of polynomial under discussion, we show that for $k>n$ or $\leq n$,

$$
\left\{z:|z|<\eta_{k}\right\} \subset\left\{z:|z|<\delta_{k}\right\} \subset \ldots \ldots \subset\{z:|z|<\beta\} \subset\{z:|z|<1+A\}
$$

In view of the class of polynomials under the present discussion Here $\delta_{k}$ is as defined in the theorem [2.1] and $\eta_{k}$ lie within and on the disc $|z| \leq \xi<1$.

To prove theorem 3, we first prove the following Lemma for $k \geq n$ :

Lemma 2: Let $p(z)=z^{n}+\sum_{j=0}^{n-1} a_{j} z^{j}$. On simplifying equation (3.1.2)

We rewrite it as:

$Q_{k}(x)=x^{k}\left(\underset{k-1}{C}-\left|a_{n-1}\right|\right) x^{k-1}+\left(\underset{k-1}{C}-\underset{k-2}{C}\left|a_{n-1}\right|-\left|a_{n-2}\right|\right) x^{k-2}+\ldots . .+\left(1-\left|a_{n-1}\right|-\left|a_{n-2}\right| \ldots . .-\left|a_{n-k+1}\right|\right) x-D=0$,

For k > n, equation (3.1.5) can be written as:

$$
Q_{k}(x)=(1+x)^{(k-1)-n}\left[\begin{array}{l}
x^{n+1}+\left(n-\left|a_{n-1}\right|\right) x^{n}+\left(\frac{n(n-1)}{2}-(n-1)\left|a_{n-1}\right|-\left|a_{n-2}\right|\right)+\ldots . .+ \\
\left(1-\left|a_{n-1}\right| \ldots .\left|a_{0}\right|\right) x-D(1+x)^{n-k+1}
\end{array}\right]
$$

Now setting:

$$
L_{n+1}(x)=x^{n+1}+\left(n-\left|a_{n-1}\right|\right) x^{n}+\ldots .+\left(1-\left|a_{n-1}\right|-\left|a_{n-2}\right| \ldots .\left|a_{0}\right|\right) x .
$$

We now give a simple proof for proving (3.16) based on mathematical induction:

Let $\mathrm{P}(\mathrm{k})$ denote the statement:

$$
Q_{k}(x)=(1+x)^{(k-1)-n}\left[L_{n+1}(x)-D(1+x)^{n-k+1}\right]
$$


Where $k=n+r, \quad r=1,2,3, \ldots \ldots \ldots . .$. and $n \in N$, the set of natural numbers

Now for $k=n+1$, from Eq. (3.1.8), we have

$$
\text { R.H.S of Eq. }(3.1 .8)=(1+x)^{(n+1-1)-n}\left[L_{n+1}(x)-D(1+x)^{n-(n+1)+1}\right]=L_{n+1}(x)-D \text {, }
$$

L.H.S of Eq. (3.1.8) $=Q_{n+1}(x)=x^{n+1}+\left(\left(\begin{array}{l}n \\ 1\end{array}\right)-\left|a_{n-1}\right|\right) x^{n}+\ldots .+\left(1-\left|a_{n-1}\right| \ldots . . .\left|a_{0}\right|\right) x-D($ from(3.1.6))

$$
=L_{n+1}(x)-D \text {, using (3.1.7) }
$$

$\mathrm{P}(\mathrm{k})$ is true for $\mathrm{n}+1$.

Now let us assume $P(k)$ is true for $k=n+r$, i.e

$$
Q_{n+r}(x)=(1+x)^{r-1}\left[L_{n+1}(x)-D(1+x)^{-r+1}\right],
$$

We now show that $P(k)$ is true for $(n+r)+1$, i.e

$$
Q_{(n+r)+1}(x)=(1+x)^{r}\left[L_{n+1}(x)-D(1+x)^{-r}\right],
$$

We now eliminate ]jfrom the Eq. (3.1.10), (3.1.11) and (3.1.12) to give:

$$
\left|\begin{array}{ccc}
Q_{n+1}(x) & 1 & 1 \\
Q_{n+r}(x) & (1+x)^{r-1} & 1 \\
Q_{n+r}(x) & (1+x)^{r} & 1
\end{array}\right|=0
$$

which on simplifying gives:

$$
\left.Q_{(n+r)+1}(x)=\frac{1}{\left[(1+x)^{r}-1\right.}\right]\left[\left\{(1+x)^{r}-1\right\} Q_{n+r}(x)-x(1+x)^{r-1} Q_{n+1}(x)\right]
$$

Since the statement that $P(k)$ is proved true for $n+1$ and assumed to be true for $\mathrm{k}=\mathrm{n}+\mathrm{r}$ as explained above, therefore by mathematical induction from Eq. (3.1.13), it is evident, that $\mathrm{P}(\mathrm{k})$ is also true for $\mathrm{k}=(\mathrm{n}+\mathrm{r})+1$. Thus statement Lemma 2 is true for $\mathrm{k}=\mathrm{n}+1,(\mathrm{n}+1)+1,(\mathrm{n}+1)$ +2 ,

Here we make use of this Lemma to prove theorem 3.1 pertaining to the polynomials having the co-efficient of the class of polynomials absolute less than unity. This process helps us in obtaining the zeros of this class of polynomials under discussion.
Now in order to obtain the bounds for the characteristic equation $Q(x)$ associated to the given polynomial $P_{n}(z)$ we note that

$$
\underset{x \rightarrow 1^{-}}{\operatorname{Lt}} Q(x)=\underset{x \rightarrow 1+}{\operatorname{Lt}} Q(x)=Q(1)
$$

In view of $Q(x)$ being a polynomial, is continuous function. We therefore, express $Q(x)$ in terms of $Q_{k}(x)$ to study the two cases of $\mathrm{k}$ such as: $k>n \& k \leq n$.

Case 1:- For k>n, from Eq. 1(b), we have:

$$
Q(1)=\underset{x \rightarrow 1}{\operatorname{Lt}} Q(x)=\underset{\eta_{k} \rightarrow 0}{\operatorname{Lt}}\left[\left(1+\eta_{k}\right)^{n}-\left|a_{n-1}\right|\left(1+\eta_{k}\right)^{n-1}-\left|a_{n-2}\right|\left(1+\eta_{k}\right)^{n-2} \ldots . .\left|a_{1}\right|\left|1+\eta_{k}\right|-\left|a_{0}\right|\right]
$$




$$
\begin{aligned}
& =\underset{\eta_{k} \rightarrow 0}{L t}\left[\left\{\eta_{k}^{n}+\left(\begin{array}{c}
n \\
1
\end{array}\right) \eta_{k}^{n-1}+\ldots+\left(\begin{array}{c}
n \\
n-1
\end{array}\right) n_{k}+1\right\}-\left|a_{n-1}\right|\left\{\eta_{k}^{n-1}+\left(\begin{array}{c}
n-1 \\
1
\end{array}\right) \eta_{k}^{n-2}+\ldots+\eta_{k}+1\right\} \ldots-\left|a_{1}\right| \eta_{k}-\left|a_{1}\right|-\left|a_{0}\right|\right] \\
& =\underset{\eta_{k} \rightarrow 0}{L t}\left[\left(\eta_{k}^{n}+\left(\begin{array}{c}
n \\
1
\end{array}\right) \eta_{k}^{n-1}+\ldots+\left(\begin{array}{c}
n \\
n-1
\end{array}\right) n_{k}+1\right)-\left|a_{n-1}\right|\left\{\eta_{k}^{n-1}+\left(\begin{array}{c}
n-1 \\
1
\end{array}\right) \eta_{k}^{n-2}+\ldots+\left(\begin{array}{c}
n \\
n-1
\end{array}\right) \eta_{k}+1\right\} \ldots-\left|a_{1}\right| \eta_{k}-\left|a_{1}\right|-\left|a_{0}\right|\right] \\
& >\underset{\eta_{k} \rightarrow 0}{L t}\left[\eta_{k}^{n}+\left(n-\left|a_{n-1}\right|\right) \eta_{k}^{n-1}+\left(\left(\begin{array}{c}
n \\
2
\end{array}\right)-\left(\begin{array}{c}
n-1 \\
2
\end{array}\right)\left|a_{n-1}\right|\right) \eta_{k}^{n-2}+\ldots .+\left(n-(n-1)\left|a_{n-1}\right|-(n-2)\left|a_{n-k}\right|-\ldots 2\left|a_{2}\right|-\left|a_{1}\right|\right)\right. \\
& \left.+\ldots\left(1-\left|a_{n-1}\right| \ldots . .\left|a_{0}\right|\right)-\frac{D}{\eta_{k}}\left(\left(1+\eta_{k}\right)^{n-(k-1)}-1\right)\right] \quad \text { Here } \frac{D}{\eta_{k}}\left(\left(1+\eta_{k}\right)^{n-(k-1)}\right) \text { is positive }
\end{aligned}
$$

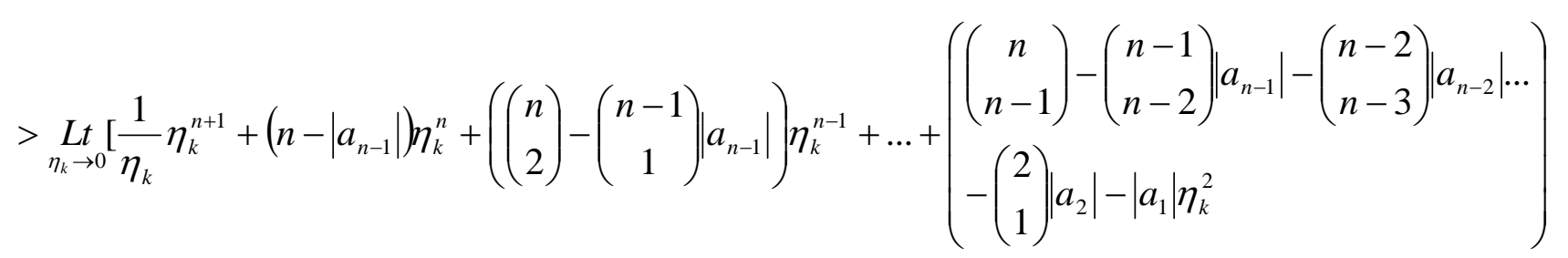

$$
\begin{aligned}
& \left.+\left(1-\left|a_{n-1}\right| \ldots . .\left|a_{0}\right|\right) \eta_{k}-D\left(1+\eta_{k}\right)^{n-(k-1)}\right] \\
& >\underset{\eta_{k} \rightarrow 0}{L t} \frac{1}{\eta_{k}}\left[L_{n+1}(x)-D\left(1+\eta_{k}\right)^{n-k+1}\right] \\
& Q(1)>\underset{\eta_{k} \rightarrow 0}{L t} \frac{1}{\eta_{k}}\left[\frac{Q_{k}\left(\eta_{k}\right)}{\left(1+\eta_{k}\right)^{(k-1)-n}}\right] \\
& \left.\Rightarrow Q(1) \geq 0 \quad \text { since } Q_{n}\left(\eta_{k}\right)=0 . \quad \text { (Since } \eta_{k} \text { as in (3.1.2) is positive root of } Q_{k}(x)=0\right)
\end{aligned}
$$

Therefore $Q(1) \geq 0 \quad \eta_{k} \rightarrow 0$ is 1 which gives $0<\xi \leq 1$.

Case 2:- Now if $k \leq n$, we have

$$
\begin{aligned}
& Q(1)=\underset{x \rightarrow 1}{\operatorname{Lt}}\left(1+\eta_{k}\right)=\underset{\eta_{k} \rightarrow 0}{\operatorname{Lt}}\left|\left(1+\eta_{k}\right)^{n}-\right| a_{n-1}\left|\left(1+\eta_{k}\right)^{n-1}-\ldots . .\right| a_{1}|| 1+\eta_{k}|-| a_{0}|| \\
& \left.\geq \underset{\eta_{k} \rightarrow 0}{\operatorname{Lt}}\left|\left(1+\eta_{k}\right)^{n}-\right| a_{n-1}\left|\left(1+\eta_{k}\right)^{n-1}-\ldots . .\right| a_{n-(k-1)} \mid\left(1+\eta_{k}\right)^{n-(k-1)}-D \eta_{k}^{2}\left(1+\eta_{k}\right)^{n-1}-\ldots . . D \eta_{k}^{2}\left(1+\eta_{k}\right)-D \eta_{k}^{2}\right] \\
& \quad \geq \underset{\eta_{k} \rightarrow 0}{L t}\left[\left(1+\eta_{k}\right)^{n}-\left|a_{n-1}\right|\left(1+\eta_{k}\right)^{n-1}-\ldots . .\left|a_{n-(k-1)}\right|\left(1+\eta_{k}\right)^{n-(k-1)}-D\left(\frac{\left(1+\eta_{k}\right)^{n-k+1}-1}{\eta_{k}}\right)\right] \\
& >\underset{\eta_{k} \rightarrow 0}{\operatorname{Lt}}\left(\frac{\left(1+\eta_{k}\right)}{\eta_{k}}\right)^{n-(k-1)}\left[\eta_{k}\left(1+\eta_{k}\right)^{k-1}-\left|a_{n-1}\right|\left(1+\eta_{k}\right)^{k-2} \eta_{k}-\left|a_{n-2}\right|\left(1+\eta_{k}\right)^{n-3} \eta_{k}-\left|a_{n-(k-1)}\right| \eta_{k}-D\right]
\end{aligned}
$$




$$
\begin{gathered}
>\underset{\eta_{k} \rightarrow 0}{L t} \frac{\left(1+\eta_{k}\right)^{n-(k-1)}}{\eta_{k}}\left[\eta_{n}^{k}+\left(k-1-\left|a_{n-1}\right| \eta_{k}^{k-1}+\left(\frac{(k-1)(k-2)}{2}-(k-2)\left|a_{n-1}\right|-\left|a_{n-2}\right|\right) \eta_{n}^{k-2}\right.\right. \\
. .+\left(1-\left|a_{n-1}\right| \ldots \ldots .\left|a_{n-(k-1)}\right| \eta_{k}-D\right] \\
>\underset{\eta_{k} \rightarrow 0}{L t} \frac{\left(1+\eta_{k}\right)}{\eta_{k}} Q_{k}\left(\eta_{k}\right) \\
>\because Q_{k}\left(\eta_{n}\right)=0
\end{gathered}
$$

In order to proof of above theorem we show that $\eta_{k}<\eta_{k-1}$. But before we give the proof, we first prove the following the recurrence relation of $Q_{k}(x)$ as given below:

Recurrence relation of $Q_{k}(x)$

Lemma $3 Q_{k}(x)$ as defined above by Eq. (3.1.7), then

$$
(1+x) Q_{k-1}(x)-Q_{k}(x)+D x=0
$$

Proof:- From Eq. (3.1.8), we have

$$
(1+x)^{n-k+1} Q_{k}(x)=L_{n+1}(x)-D(1+x)^{n-k+1}
$$

Replacing k by k-1 from eq. (3.1.8) we have

$$
(1+x)^{n-k+2} Q_{k-1}(x)=L_{n+1}(x)-D(1+x)^{n-k+2}
$$

Now eliminating $L_{n+1}(x)$ from above two equations, we have

$$
(1+x)^{n-k+1}\left[Q_{k}(x)-(1+x) Q_{k-1}(n)\right]=D(1+x)^{n-k+1}[(1+x)-1]
$$

This prove recurrence relation given by (4.1.1)

Proof of the Theorem:-

Since $\eta_{k-1}$ is the root of $Q_{k-1}\left(\eta_{k-1}\right)=0$

Now put $x=\eta_{k-1}$ in the (4.1.1) is

$$
\left(1+\eta_{k-1}\right) Q_{k-1}\left(1+\eta_{k-1}\right)-Q_{k}\left(1+\eta_{k-1}\right)+\eta_{k-1}=0
$$

Where D \& $\eta_{k-1}$ are

Now

$$
\begin{gathered}
Q_{k}\left(\eta_{k-1}\right)=Q_{R}\left(\eta_{k-1}\right)-\left(1+\eta_{k-1}\right) Q_{k-1}\left(\eta_{k-1}\right) \\
=D \eta_{k-1}, \therefore D=\sum\left|a_{j}\right|, 0 \leq j \leq n-1 .
\end{gathered}
$$

Hence $Q_{R}\left(\eta_{k-1}\right)>0$, where as $Q_{R}\left(\eta_{k}\right)=0$. This implies $\eta_{k} \leq \eta_{k-1}$ this complete the proof of the theorem.

\subsection{Some Examples based on the above Theorem:}

Corresponding to the polynomial $P_{n} z$ we solve the so called characteristic equation $Q_{k}(x)=0$. The output of this result is as given below in the following tables. In the $\mathrm{I}^{\text {st }}$ column we have the degree from 1 to $\mathrm{k}$ of the algebraic equation $Q_{k}(x)=0$ in the second column we have the corresponding bounds $1+\delta_{k}$ for the radius of the circle that contains all the zeros of the polynomials and in the $3^{\text {rd }}$

\begin{tabular}{|c|c|c|c|}
\hline \multirow{2}{*}{$\begin{array}{l}\text { Values } \\
\text { of } \\
\text { K }\end{array}$} & Their Bound & Our Bound & \multirow{2}{*}{$\begin{array}{l}\text { Over estimate of } \\
\text { error }\end{array}$} \\
\hline & $|z| \leq 1+\delta_{k}$ (of others) & $\left.|z| \leq \max \} 1, \eta_{k}\right\}=1, k \geq 3,:$ & \\
\hline 1 & 1.25000000 & $\{1, .75\}$ & \multirow{3}{*}{$\begin{array}{l}\text { For } \mathrm{k}=10, \text { the } \% \\
\text { corresponding to the } \\
\text { bounds obtained by } \mathrm{C} \text {. }\end{array}$} \\
\hline 2 & 2.00000000 & $\{1,1.31872929=1.3872929$ & \\
\hline 3 & 1.21972895 & $\{1, .43861017\}$ & \\
\hline
\end{tabular}
column we tabulate the present bounds by the present class of polynomials corresponding to the bounds $\max \left(1, \eta_{k}\right)$. On comparison we show that the present bounds obtained are the infimum (lesser of lub) enclosing the respective zeros of the polynomials as compared to the lub of C Affane Aji. The output of these example is as given below:

\section{Example 1:}

Let $p(z)=z^{5}+\frac{1}{4}\left(z^{4}+z^{2}+1\right)$, true bound $=$ 0.91119850 


\begin{tabular}{|l|l|l|l|}
\hline 4 & 1.21372896 & $\{1.38706535\}$ & $\begin{array}{l}\text { Affane. Aji. et al [1] } \\
\text { over the present } \\
\text { estimate, errors by a } \\
\text { factor 2.6. }\end{array}$ \\
\cline { 1 - 3 } & 1.9237991 & $\{1.33438766\}$ & \\
\hline 7 & 1.1802918 & $\{1.29419863\}$ & \\
\hline 9 & 1.17815386 & $\{1.27942836\}$ & \\
\hline 10 & 1.16181430 & $\{1, .25511065\}$ & \\
\hline
\end{tabular}

\section{Example 2:}

Let $p(z)=z^{15}+\frac{1}{2}\left(z^{13}+z^{12}-z^{10}\right)-\frac{1}{3} z^{6}+\frac{1}{5} z^{3}+\frac{1}{3} z-\frac{1}{4}$,

\begin{tabular}{|c|c|c|c|}
\hline \multirow{2}{*}{$\begin{array}{l}\text { Values } \\
\text { of } \\
\text { K }\end{array}$} & Their Bound & Our Bound & \multirow[t]{2}{*}{ Over estimate of error } \\
\hline & $|z| \leq 1+\delta_{k}($ of others $)$ & $\left.|z| \leq \max \} 1, \eta_{k}\right\}=1, k \geq 3,:$ & \\
\hline 1 & 1.50000000 & $\{1,2.616\}=2.6166666$ & \multirow{10}{*}{$\begin{array}{l}\text { Over estimation factor of } \\
\text { previous authors } \\
\text { corresponding to } \mathrm{k}=10 \text {, the } \\
\text { bounds obtained C. Affane. } \\
\text { Aji. et al [1] over estimate } \\
\text { the present bounds at least } \\
\text { by a factor } 5.8 \text { and in view } \\
\text { of error is } 0.31393132 \text { : } \\
0.053474\end{array}$} \\
\hline 2 & 1.36602593 & $\{1,2.19292693\}=2.1929269$ & \\
\hline 3 & 1.36602542 & $\{1, .887149531\}$ & \\
\hline 4 & 1.36602542 & $\{1, .71292043\}$ & \\
\hline 5 & 1.32471797 & $\{1, .51521635\}$ & \\
\hline 6 & 1.30080360 & $\{1, .51451182\}$ & \\
\hline 7 & 1.30080357 & $\{1, .47310513\}$ & \\
\hline 8 & 1.29996768 & $\{1, .46837437\}$ & \\
\hline 9 & 1.27138251 & $\{1, .40011427\}$ & \\
\hline 10 & 1.26045701 & $\{1, .37009931\}$ & \\
\hline
\end{tabular}

\section{Example 3:}

Let $p(z)=z^{25}+\frac{1}{5} z^{23}-\frac{1}{8}\left(z^{21}+z^{16}-z^{15}\right)-\frac{1}{10}\left(z^{10}-z^{6}+z^{3}\right)+\frac{1}{50} z^{2}-\frac{1}{10}=0$, true bound $=0.9225556$

\begin{tabular}{|l|l|l|l|}
\hline \multirow{2}{*}{$\begin{array}{l}\text { Values } \\
\text { of }\end{array}$} & Their Bound & Our Bound & Over estimate of error \\
\cline { 2 - 3 } & $|z| \leq 1+\delta_{k}$ (of others) & $|z| \leq \max \left\{1, \eta_{k}\right\}=\forall k, k \neq 2$ & \\
\hline 1 & 1.20000000 & $\{1,0.995\}$ & $\begin{array}{l}\text { Over estimation of previous } \\
\text { authors[1] corresponding to } \\
k=10 \text { by factor 2.4. In view } \\
\text { of the rel. errors of other } \\
\text { authors and present authors as: }\end{array}$ \\
\hline 3 & 1.7082014 & $\{1,1.657956\}$ & \multirow{2}{*}{$0.18775156: 0.077444}$. \\
& 1.708204 & $\{1, .49147788\}$ & \\
\hline 5 & 1.15340459 & $\{1, .44147179\}$ & \\
\hline 7 & 1.14820807 & $\{1, .381310340\}$ & \\
\hline 9 & 1.14857797 & $\{1.29817784\}$ & \\
\hline 10 & 1.12732975 & $\{1, .27032855\}$ & \\
\hline
\end{tabular}

\section{Example 4:}

Let

$$
p(z)=z^{35}+\frac{1}{20} z^{35}\left(5 z^{34}+10 z^{32}+4 z^{31}+4 z^{30}\right)+\frac{i}{140}\left(20 z^{22}+5 z^{21}+10 z^{19}+4 z^{18}+4 z^{17}+20 z^{5}\right.
$$

$\left.+\frac{35}{6} z^{4}+10 z^{2}+4 z+4\right)$, true bound $=.89920688$

\begin{tabular}{|c|c|c|c|}
\hline \multirow{2}{*}{$\begin{array}{l}\text { Values } \\
\text { of } \\
\mathbf{K}\end{array}$} & Their Bound & Our Bound & \multirow[t]{2}{*}{ Over estimate of error } \\
\hline & $|z| \leq 1+\delta_{k}($ of others $)$ & $|z| \leq \max \left\{1, \eta_{k}\right\}=1, k \geq 3$ & \\
\hline 1 & 1.50000000 & $\{1,1.77\}=1.7714205$ & \multirow{3}{*}{$\begin{array}{l}\text { Over estimate of error factors } \\
\text { corresponding to } \mathrm{k}=10 \text {,the } \\
\text { bounds obtained } \mathrm{C} \text {. Affane. }\end{array}$} \\
\hline 2 & 1.7539049 & $\{1,1.75725359\}=1.75725359$ & \\
\hline 3 & 1.2670351 & $\{1, .57764816\}$ & \\
\hline
\end{tabular}




\begin{tabular}{|c|c|c|c|}
\hline 4 & 1.34667841 & $\{1, .51961333\}$ & \multirow{7}{*}{$\begin{array}{l}\text { Aji. et al [1] overstate the } \\
\text { present bounds or least by a } \\
\text { factor } 3 \text {. In view of negative } \\
\text { error of other authors and the } \\
\text { present as } 0.32286685 \text { : } \\
0.10079\end{array}$} \\
\hline 5 & 1.31837386 & $\{1, .51664084\}$ & \\
\hline 6 & 1.29831222 & $\{1,39623964\}$ & \\
\hline 7 & 1.27480981 & $\{1, .38846096\}$ & \\
\hline 8 & 1.25438958 & $\{1, .36794695\}$ & \\
\hline 9 & 1.23839181 & $\{1, .34069264\}$ & \\
\hline 10 & 1.22287373 & $\{1, .3150619\}$ & \\
\hline
\end{tabular}

From the above table we note the upper bounds obtained by C. Affane.Aji. et al [1] over estimate the corresponding bounds due to the present investigation in all the cases at least by a factor 2.5 and thereby is confirm that in all the cases $k \geq 3$, unit disc is the least upper bounds enclosing the zeros of the class of polynomials under the present discussion.

\section{REFERENCES}

[1] C. Affane-Aji, N.Agarwal \& N.K. Govil, Mathematical and Computer Modelling.

[2] A.L. Canchy, Exercises de mathmatiques, IV Annee de Bure Freres, Paris, 1829.

[3] B. Datt, N.K. Govil, on the location of zeros of polynomial, J. Approximation theory 24(1978), 7882.

[4] K. F. Gauss, Beitrage zur Theorie der algebraisshen Gleichungen, Abh. Ges. Wiss. Gottingen 4(1850) Ges. Werkr. Vol 3, pp73-102.

[5] V.K. Jain on Cauchy's bounds for zeros of polynomials Turkish.J. Math. 30(2006), 95-100.

[6] A. Joyal, G. Labelle, Q.I. Rehman on location of zeros of polynomials Canada.math.bull. 10(1967), 53-63.

[7] M. Marden, Geometry of polynomials, Amer. Math Society, Math surveys Vol. 3, Amer Math society, providence, R.I, 1966.

[8] Z. Rabinstein, Some results in the location the zeros of linear combinations of polynomials, Trans. Amer. Math. Soc. 116(1965), 1-8.

[9] Y.J. Sun, J.G. Hsieh, Anote on circular bounds of polynomials of zeros, IEEE Trans, Circuits system I, Regul pap 43(1996), 476-478.

[10] M.S. Zilric, L.M. Roytman, P.L. Combettes, M.N. Swamy, A bound for the zeros of polynomials, IEEE Trans circuits systemI, 39(1992), 476-478. 